\title{
ARTICLE
}

\section{Improved CT-based voxel phantom generation for MCNP Monte Carlo}

\author{
Michael Speiser $^{\mathrm{a}^{*}}$ and John DeMarco ${ }^{\mathrm{b}}$ \\ ${ }^{a}$ University of Texas Southwestern Medical Center, Dallas, TX 75390-8542; ${ }^{b}$ University of California, Los Angeles, CA 90095-6951
}

\begin{abstract}
A graphic user interface has been developed to perform the basic and advanced functions required to create CT-based, voxelized patent models for MCNP and MCNPX Monte Carlo simulations. Additional flexibility is enabled in selective tally definition, lattice structure limits, and conversion of CT number to materials. Setting lattice structure limits can reduce or obviate the need for excessive sub-sampling and improve efficiency while preserving model resolution. The patient model, tally definition, and subsequent simulation output are all incorporated in a post-processing routine that enables comparative analysis between calculated and measured datasets. Judicious application of these developments can greatly decrease the time required to achieve a given uncertainty while preserving model detail; use of these tools typically results in reduction of simulation times by over $60 \%$.
\end{abstract}

Keywords: Monte Carlo; MCNP; radiotherapy

\section{Introduction}

CT-based, voxelized patient models are often used in Monte Carlo investigations. In order to automate the basic functions required to create a voxelized patient model from a CT dataset for use with the Los Alamos Monte Carlo N-Particle (MCNP) program, DeMarco et al developed the "RTMCNP Preprocessor" [1-3].

While CT-based patient models can preserve detail, radiation transport simulation through a high-resolution lattice can be prohibitively resource intensive. This work builds on the basic functionality of the RTMCNP program in order to enable an investigator to optimize the model's spatial resolution, lattice size, CT-to-material conversion, tally definition, and variance reduction applications. A program with a graphic user interface called the MCNP_GUI has been developed to perform and visualize these functions while generating voxelized Monte Carlo models in order to increase simulation efficiency while maintaining model detail and accuracy.

\section{Methods}

\subsection{Basic function and data visualization}

The MCNP_GUI program is written in Information Data Language (IDL) and can be compiled for use as a standalone executable program. The MCNP_GUI allows the user to browse for a CT dataset using familiar windows interfaces. Once the CT dataset has been

*Corresponding author. Email: mike.speiser@gmail.com identified, MCNP GUI searches the DICOM tags for the information needed to convert voxel values into Hounsfield Units (HU's) as well as geometric information such as slice thickness and voxel size. The lattice is displayed in an intuitive CT-Scan viewing format, allowing the user to scroll through and visualize the dataset as shown in Figure 1.

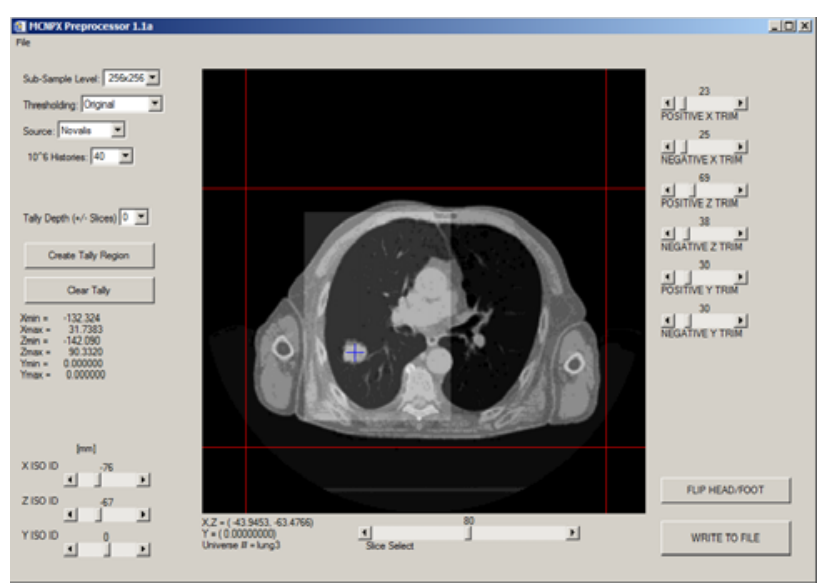

Figure 1. MCNP_GUI image showing interface layout and functionality.

\subsection{Material Conversion}

Each HU is translated into a material-density combination using one of several customizable lookup tables. By default, MCNP_GUI converts each HU into one of seventeen material-density combination using a lookup table as described by DeMarco et al. and recreated in Table 1 [3]. Additionally, the user can 
select conversion tables based on the subject of the scan (e.g. for QA phantoms, several tables exist to translate HU's into QA phantom materials as provided by a specific manufacturer). Mouse-over functions display the results of the material conversion.

Table 1. Default Hounsfield Unit to Material lookup table.

\begin{tabular}{|c|c|c|c|}
\hline HU range & Material & $\begin{array}{c}\text { Composition } \\
(\% \mathrm{Z})\end{array}$ & $\begin{array}{l}\text { Density } \\
\left(\mathrm{g} / \mathrm{cm}^{3}\right)\end{array}$ \\
\hline $\mathrm{HU}<-954$ & air & $\begin{array}{c}75.5 \mathrm{~N}, 23.2 \mathrm{O} \\
1.3 \mathrm{Ar}\end{array}$ & 0.0012 \\
\hline$-954 \leq \mathrm{HU}<-824$ & \multirow{5}{*}{ lung } & \multirow{5}{*}{$\begin{array}{c}10.4 \mathrm{C}, 10.3 \mathrm{H} \\
2.9 \mathrm{~N}, 76.4 \mathrm{O}\end{array}$} & 0.048 \\
\hline$-824 \leq \mathrm{HU}<-674$ & & & 0.1254 \\
\hline$-674 \leq \mathrm{HU}<-524$ & & & 0.2978 \\
\hline$-524 \leq \mathrm{HU}<-374$ & & & 0.4721 \\
\hline$-374 \leq \mathrm{HU}<-224$ & & & 0.6455 \\
\hline$-224 \leq \mathrm{HU}<-159$ & \multirow{3}{*}{ fat } & \multirow{3}{*}{$\begin{array}{c}63.7 \mathrm{C}, 11.9 \mathrm{H} \\
23.2 \mathrm{O}, 0.8 \mathrm{~N}\end{array}$} & 0.85 \\
\hline$-195 \leq \mathrm{HU}<-94$ & & & 0.925 \\
\hline$-94 \leq \mathrm{HU}<-29$ & & & 0.98 \\
\hline$-29 \leq \mathrm{HU}<19$ & water & $11.1 \mathrm{H}, 88.9 \mathrm{O}$ & 1.0 \\
\hline $19 \leq \mathrm{HU}<29$ & \multirow{3}{*}{ muscle } & \multirow{3}{*}{$\begin{array}{c}10.8 \mathrm{C}, 10.1 \mathrm{H} \\
2.8 \mathrm{~N}, 73.6 \mathrm{O}\end{array}$} & 1.06 \\
\hline $29 \leq \mathrm{HU}<76$ & & & 1.14 \\
\hline $76 \leq \mathrm{HU}<256$ & & & 1.26 \\
\hline $256 \leq \mathrm{HU}<436$ & \multirow{4}{*}{ bone } & \multirow{4}{*}{$\begin{array}{c}14.3 \mathrm{C}, 20.8 \\
\mathrm{Ca}, 4.86 \mathrm{H}, \\
4.16 \mathrm{C}, 44.4 \mathrm{O}, \\
10.6 \mathrm{Si}\end{array}$} & 1.48 \\
\hline $436 \leq \mathrm{HU}<616$ & & & 1.68 \\
\hline $616 \leq \mathrm{HU}<796$ & & & 1.89 \\
\hline $796 \leq \mathrm{HU}$ & & & 2.1 \\
\hline
\end{tabular}

\subsection{Resampling source data}

A typical CT-Scan has sub-millimeter spatial resolution that may not be warranted for the intended patient model and simulation. Sub-sampling functions allow the user to repeatedly sub-sample from the original CT dataset and visualize the result. Visual inspection of the sub-sampled dataset allows the user to assess if model detail is sufficiently preserved and if the resulting material conversion is still appropriate.

\subsection{Selective tally definition}

MCNP_GUI allows the user to define a Rectangular Mesh Tally (RMESH) over an arbitrary region of interest. In limiting the extent of the tally, the simulation speed is improved.

The RMESH is a type T (or "Total") tally and records all energy deposited in its elements in units of $\mathrm{MeV} / \mathrm{cm}^{3} /$ starting particle. In order to convert this energy deposition into dose, MCNP_GUI automatically defines the RMESH elements as geometrically equivalent and collocated with the user-selected elements of the final lattice. When the input file is generated, a density matrix corresponding to the same lattice elements covered by the RMESH tally is also created. Once the simulation is completed, the energy deposition matrix is divided by the density matrix to convert tally results to dose as seen in Eq. (1).

$$
\operatorname{dose}_{x, y, z}=E_{x, y, z}\left(\mathrm{MeV} / \mathrm{cm}^{3}\right) \div \rho\left(\mathrm{g} / \mathrm{cm}^{3}\right)
$$

\subsection{Lattice trimming}

A single slice of a CT dataset consists of $512 \times 512$ (or 262,144) elements. Individual slices are often sub-sampled (e.g. from $512 \times 512$ to $128 \times 128$ elements per slice) to improve simulation efficiency by reducing the number of surfaces and tallying in larger volumes. However, sub-sampling also causes a loss of resolution. One solution that can greatly improve the efficiency of a simulation while preserving a given resolution is to truncate the lattice to contain only the patient model on interest (e.g. removing layers of the lattice that represent air or areas of the model far removed from any source particle interactions). This process has been implemented in the MCNP_GUI.

An example screenshot of this process is shown in Figure 2. The four red lines surrounding the lattice display the user-defined limits for the new lattice. The original CT scan consists of 160 slices and has been already sub-sampled 256x256 elements per slice. Following that, the lattice is trimmed 23 and 25 layers in the x-dimension, 69 and 38 layers in the z-dimension, and 30 and 30 slices in the y-dimension. This lattice trimming further reduces the sub-sampled scan from $10,485,760$ to $3,099,200$ elements (or by approximately $70 \%$ ) without excessive sub-sampling and preserving model detail.

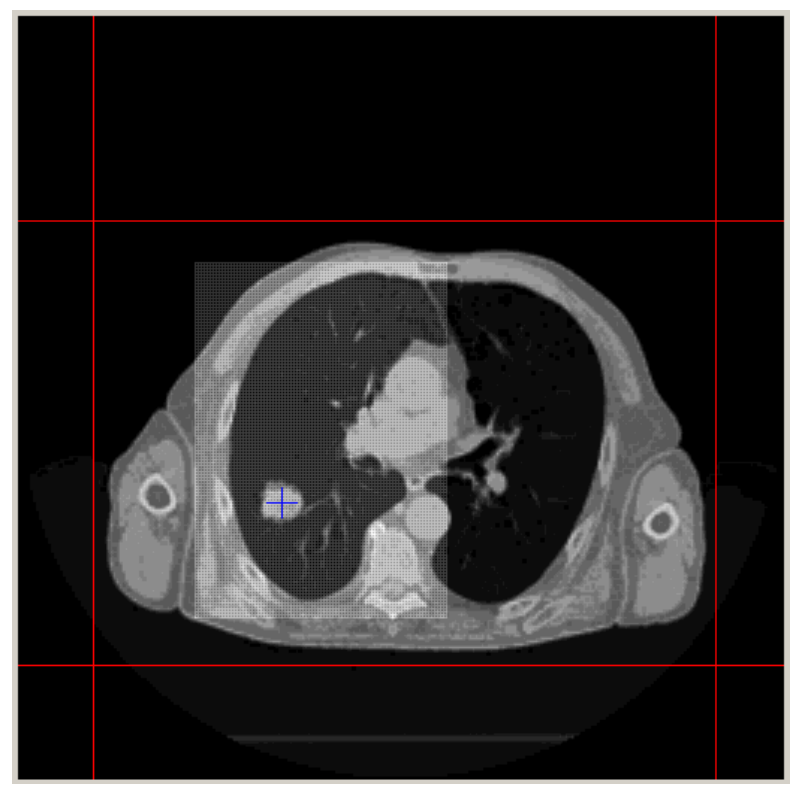

Figure 2. Detail of MCNP_GUI viewing window, displaying lattice, selective tally definition (mesh overlaying patient's right lung), lattice truncation (four red lines surrounding body), and isocenter placement (blue crosshair in tumor volume). 
An isocenter definition function allows the user to visualize and move the isocenter within the lattice geometry. This location is then available for use with any custom source definition. In the example shown, the isocenter coordinates are used to shift the virtual source planes for custom source definition of the Novalis linac.

Finally, when the MCNP (or MCNPX) formatted input file is generated, the MCNP_GUI outputs a matrix for all CT slices collocated with tallies. This allows visualizing the simulated dose distribution correctly overlaying the corresponding patient images.

\subsection{Post-processing and visualizing simulation results}

\subsubsection{Normalization and display}

An additional program has been written that pairs with the MCNP GUI for post-processing, analysis, and display. Following the conclusion of a simulation, a post-processing routine gathers information from the MCNP/MCNPX output files as well as the density matrix and CT images generated by the MCNP GUI. The energy deposition matrix is divided by the associated lattice density matrix, and the resulting dose matrix is normalized to its maximum value that has an acceptable user-defined R-value. Simulated and calculated dose distributions can easily be displayed over the corresponding CT image; the simulation prepared in Figures 1 and 2 is shown in Figure 3.

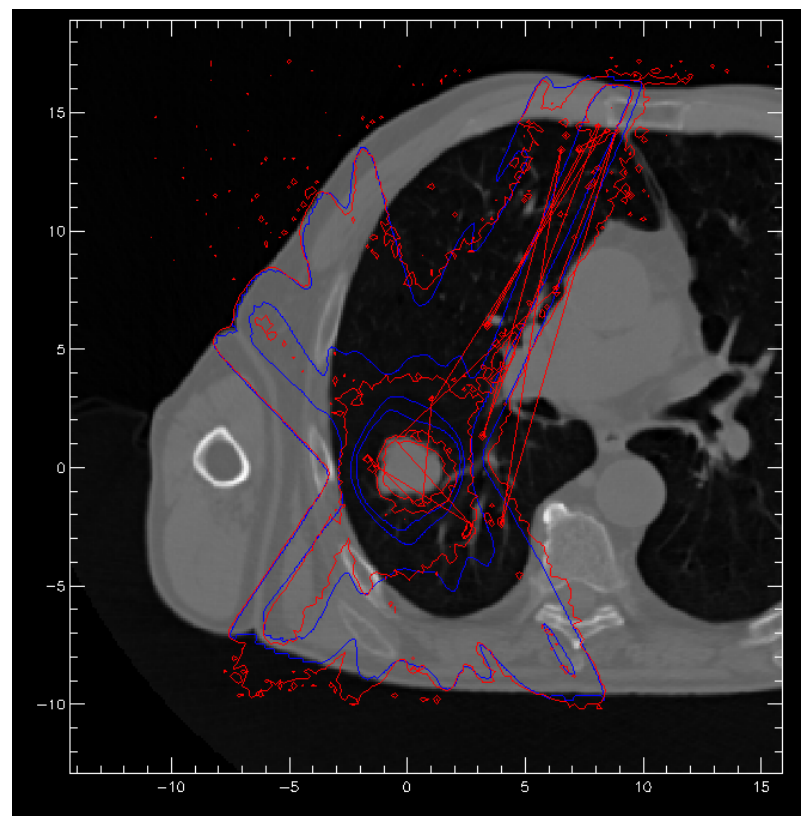

Figure 3. Overlay of simulated (red) and calculated (blue) normalized dose distributions using the post-processing software.

\subsubsection{Comparative analysis}

Further, the post-processing software is capable of performing comparative analysis between the simulation results and other datasets (e.g. calculated or measured dose distributions). This method of comparative analysis is called gamma analysis, and it is routinely used in radiotherapy.[4,5] Using gamma analysis to analyze results provides a common reference to assess clinical relevance of disagreement.

\section{Results}

The patient model, tally definition, and subsequent simulation output are all incorporated in a post-processing routine that enables comparative analysis between calculated and measured datasets. Judicious application of these developments can greatly decrease the time required to achieve a given uncertainty while preserving model detail; use of these tools typically results in reduction of simulation times by over $60 \%$ to achieve the same uncertainty.[6]

\section{Conclusion}

Despite advances in computer hardware and programming, Monte Carlo remains resource intensive and largely restricted to the domain of the expert. The MCNP_GUI enables generation of accurate and efficient CT-based MCNP/MCNPX patient models. Simulation results are easily normalized and interpreted in clinical radiotherapy terms. The MCNP GUI integrates many advanced Monte Carlo functions in an intuitive and accessible way, providing a powerful tool for the Monte Carlo investigator.

\section{Acknowledgements}

The authors wish to thank Adam Kesner, Ph.D. for his assistance in graphic user interface development.

\section{References}

[1] F. Briesmeister, MCNP-A General Monte Carlo $N$-Particle Transport Code, Version 4A, Los Alamos National Laboratory report, LA-12625, (1993).

[2] T. Goorley, M. James, T. Booth et al., Initial MCNP6 release overview, Nucl. Tech. 180 (2012), pp. 298-315.

[3] J. DeMarco, T. Solberg and J. Smathers, A CT-based Monte Carlo simulation tool for dosimetry planning and analysis, Med. Phys. 25 (1998), pp. 1-11.

[4] D. Low, W. Harms, S. Mutic and J. Purdy, A technique for the quantitative evaluation of dose distributions, Med. Phys. 25 (5) (1998), pp. 656-661.

[5] N. Agazaryan, T. Solberg and J. DeMarco, Patient specific quality assurance for the delivery of intensity modulated radiotherapy, J. Appl. Clin. Med. Phys. 4 (2003), pp. 40-50.

[6] M. Speiser, J. DeMarco, N. Agazaryan, I. Chetty, T. Solberg and A. Kesner, Development and validation of an MCNPX Monte Carlo IMRT source model with optimized radiotherapy interface and comparative analysis software, Med. Phys. 34 (2007), p. 2435 